\title{
Transmon in a semi-infinite high-impedance transmission line: Appearance of cavity modes and Rabi oscillations
}

\author{
E. Wiegand $\odot,{ }^{1, *}$ B. Rousseaux $\odot,{ }^{2}$ and G. Johansson $\odot^{1}$ \\ ${ }^{1}$ Applied Quantum Physics Laboratory, Department of Microtechnology and Nanoscience-MC2, Chalmers University of Technology, \\ 41296 Göteborg, Sweden \\ ${ }^{2}$ Laboratoire de Physique de l'École Normale Supérieure, ENS, Université PSL, CNRS, Sorbonne Université, Université de Paris,
} 75005 Paris, France

(Received 8 December 2020; accepted 11 March 2021; published 1 April 2021)

\begin{abstract}
In this paper, we investigate the dynamics of a single superconducting artificial atom capacitively coupled to a transmission line with a characteristic impedance comparable to or larger than the quantum resistance. In this regime, microwaves are reflected from the atom also at frequencies far from the atom's transition frequency. Adding a single mirror in the transmission line then creates cavity modes between the atom and the mirror. Investigating the spontaneous emission from the atom, we then find Rabi oscillations, where the energy oscillates between the atom and one of the cavity modes.
\end{abstract}

DOI: 10.1103/PhysRevResearch.3.023003

\section{INTRODUCTION}

In the past two decades, circuit quantum electrodynamics (circuit QED) has become a field of growing interest for quantum information processing and also to realize new regimes in quantum optics [1-11]. The restriction to one-dimensional (1D) waveguides in circuit and waveguide QED enhances directionality and reduces losses and therefore has a great advantage over higher-dimensional systems to reach strongand ultrastrong-coupling regimes [12-22]. A typical circuit QED setup consists of a superconducting qubit coupled to a 1D transmission line (TL) [2,6-8,23]. Superconducting qubits are artificial atoms built with a nonlinear Josephson Junction (JJ) that creates an anharmonic energy spectrum [9]. There are different kinds of superconducting qubits, such as flux qubits, phase qubits, and charge qubits $[8,24]$. A 1D transmission line can be modeled by coupled $L C$ oscillators, each having a characteristic impedance of $Z_{0}=\sqrt{L_{0} / C_{0}} \approx 50 \Omega$, smaller than the quantum resistance $R_{Q}=\hbar /(2 e)^{2} \approx 1.0 \mathrm{k} \Omega$. But recent studies showed that it is possible to reach impedances comparable to the quantum resistance or higher [25-31]. This can be realized by building circuits made of arrays of JJs [27,28,30-34] or high-kinetic-inductance materials, called superinductors [25,26,29,35-37]. High-impedance JJ arrays and superinductors are, for example, used in the fluxonium qubit [38-41], which has reduced charge noise sensitivity and can have relaxation times up to milliseconds $[40,42]$. This also has an advantage for metrology since the charge noise insensitivity makes it possible to measure the current very accurately

\footnotetext{
*wiegand@chalmers.se

Published by the American Physical Society under the terms of the Creative Commons Attribution 4.0 International license. Further distribution of this work must maintain attribution to the author(s) and the published article's title, journal citation, and DOI.
}

[43]. Furthermore, high-impedance resonators make it possible for light-matter interaction to reach strong-coupling regimes due to strong coupling to vacuum fluctuations [44].

In this article, we investigate the spontaneous emission of a transmon [45] capacitively coupled to a 1D TL that is shorted at one end. This system is known as an "atom in front of a mirror" [46-51]. Instead of using a Markovian master equation approach, we are taking the photon traveling time fully into account, making the dynamics nontrivial [14,5261]. Furthermore, we explore the above-mentioned regime of a TL impedance $Z_{0}$ exceeding the impedance of the transmon $Z_{J}=R_{Q} \sqrt{2 E_{C} / E_{J}}, Z_{0} \gg Z_{J}$. We find that the system behaves qualitatively differently compared to the well-studied low-impedance regime $Z_{0} \ll Z_{J}$. The atom reflects strongly at all frequencies, except its transition frequency. Together with the mirror, it thus forms a cavity, and when the transition frequency is close to a cavity mode, we find a vacuum Rabi splitting, resulting in Rabi oscillations in the spontaneous emission. In this regime, all dynamical timescales are independent of the coupling capacitance and instead depend on the intrinsic transmon capacitance and the TL impedance.

Other cavity-free systems that show Rabi splitting and Rabi oscillations have been found with giant atoms [62], e.g., an artificial atom coupled to surface acoustic waves [63]. Systems involving atoms that act like mirrors were also studied experimentally with a single trapped cold ion in front of a dielectric mirror [64] and exploration of collective dark states of strongly coupled qubits [65].

\section{CIRCUIT-QED MODEL}

Our system consists of a transmon qubit capacitively coupled to a semi-infinite $1 \mathrm{D} T L$ at a distance $L$ from its grounded end (see Fig. 1). The transmon qubit consists of a superconducting anharmonic $L C$ oscillator, where the inductive $(L)$ element is formed by a JJ with characteristic energy $E_{J}$ in 


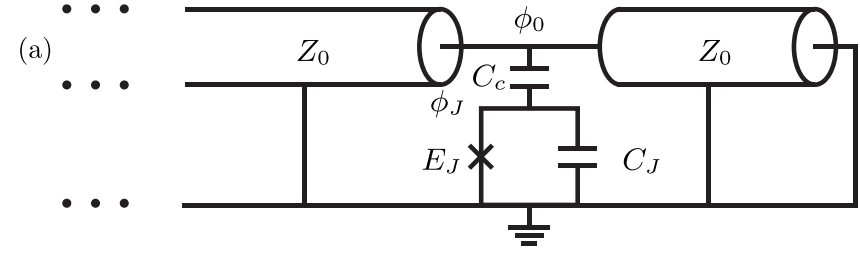

(b)
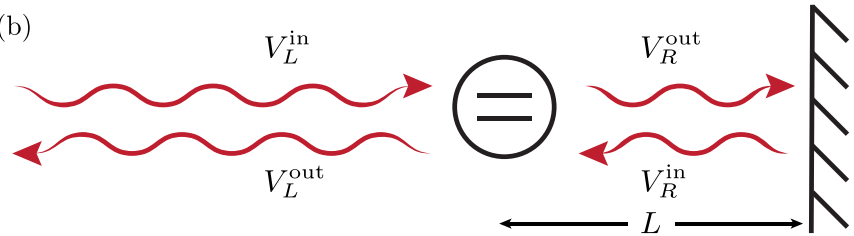

FIG. 1. (a) Circuit model of a transmon coupled through the coupling capacitance $C_{c}$ to a semi-infinite 1D TL with impedance $Z_{0}$. The Josephson energy, flux, and capacitance of the transmon are denoted by $E_{J}, \phi_{J}$, and $C_{J}$. The flux on the coupling capacitance $C_{c}$ is denoted by $\phi_{0}$, with the corresponding voltage $V_{0}=\dot{\phi}_{0}$. (b) Sketch of the system depicting an atom in front of a mirror coupled to incoming and outgoing microwave fields to the left and right characterized by their respective voltages $V_{L / R}^{\text {in/out }}$, respectively, at the transmon. The mirror couples the fields to the right $V_{R}^{\text {in }}(t)=-V_{R}^{\text {out }}(t-T)$, introducing the time of propagation to the mirror and back $T=2 L / v$.

parallel with a capacitor $C$ with capacitance $C_{J}$. The sinusoidal current-phase relation of the $\mathrm{JJ}$ makes the energy spectrum of the transmon qubit anharmonic, allowing for excitation with a single microwave photon using standard harmonic microwave sources. The transmon is capacitively coupled to a microwave $\mathrm{TL}$, characterized by its inductance per unit length $L_{0}$ and capacitance per unit length $C_{0}$. The velocity and characteristic impedance of the electromagnetic field inside the TL are given by $v=1 / \sqrt{L_{0} C_{0}}$ and $Z_{0}=\sqrt{L_{0} / C_{0}}$, respectively. Using the standard circuit quantization procedure [61,66,67], we can derive the Heisenberg equations of motion for the charge $p_{0}(t)$ on the coupling capacitor $C_{c}$, the charge $p_{J}(t)$ on $C_{J}$, and its conjugate flux $\phi_{J}(t)$, giving the phase difference $2 e \phi_{J}(t) / \hbar$ over the JJ. Denoting the operators for the voltages of the incoming and outgoing microwave fields to the left and right of the transmon as $V_{L / R}^{\mathrm{in} / \mathrm{out}}(t)$ [see Fig. 1(b)], respectively, these equations are

$$
\begin{gathered}
\partial_{t} \phi_{J}(t)=\frac{1}{C_{J}}\left[p_{J}(t)+p_{0}(t)\right], \\
\partial_{t} p_{J}(t)=-E_{J} \frac{2 e}{\hbar} \sin \left[\frac{2 e}{\hbar} \phi_{J}(t)\right] \approx-\frac{\phi_{J}(t)}{L_{J}}, \\
\partial_{t} p_{0}(t)=\frac{2 p_{0}(t)}{Z_{0} C_{\Sigma}}+\frac{2 p_{J}(t)}{Z_{0} C_{J}}-\frac{2}{Z_{0}}\left[V_{L}^{\text {in }}(t)+V_{R}^{\text {in }}(t)\right], \\
V_{L / R}^{\text {out }}(t)=V_{R / L}^{\text {in }}(t)-\frac{Z_{0}}{2} \partial_{t} p_{0}(t),
\end{gathered}
$$

where we denoted the capacitance to the ground seen by the coupling node as $C_{\Sigma}=C_{c} C_{J} /\left(C_{c}+C_{J}\right)$ and, in the second equation, we introduced the Josephson inductance $L_{J}=$ $\hbar^{2} / 4 e^{2} E_{J}$, which describes the linearized dynamics of the Josephson junction. This approximation is obviously good in the weak-excitation regime $\left|\phi_{J}(t)\right|<\hbar / 2 e$ and will also be sufficient to describe the spontaneous emission, where the

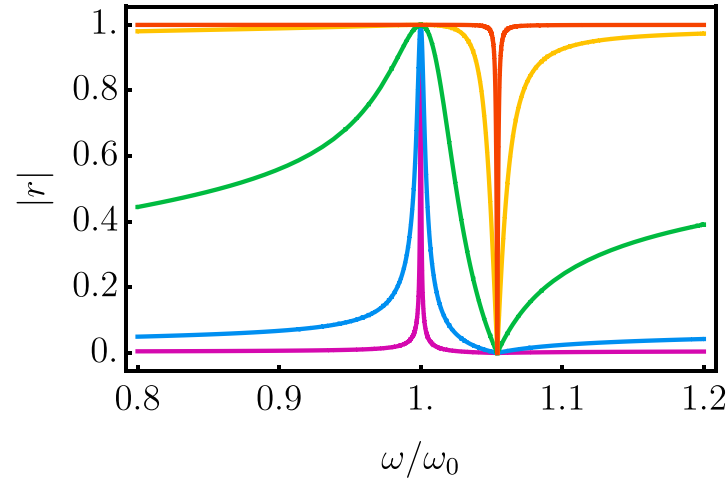

FIG. 2. Reflection of a transmon in an open TL for different ratios of the TL and qubit impedance $Z_{0} / Z_{J}$. The curves show the reflection for $\frac{C_{c}}{C_{c}+C_{J}}=0.1$ and $Z_{0} / Z_{J}=0.1$ (purple), $Z_{0} / Z_{J}=$ 1 (blue), $Z_{0} / Z_{J}=10$ (green), $Z_{0} / Z_{J}=100$ (yellow), and $Z_{0} / Z_{J}=$ 1000 (red) from low to high TL impedance. For low impedance, the qubit reflects only at its low-impedance frequency $\omega_{0}$, but for high impedance, it reflects everywhere but its bare frequency $\omega_{J}$.

transmon is initially excited by a single microwave photon. Here, we note that the equations of motion are linear and thus could be understood from classical dynamics.

\section{REFLECTION}

\section{A. Open TL}

To characterize how the behavior of the system changes when we increase the TL impedance $Z_{0}$, we first investigate the reflection of microwaves from the transmon coupled to an open TL, i.e., without a mirror. Since the equations of motion are linear, we can express the reflected field operator $V_{L}^{\text {out }}$ in terms of the incoming probe field operator $V_{L}^{\text {in }}$ by Fourier transforming the equations of motion (1)-(4), assuming no incoming field from the right $\left(V_{R}^{\mathrm{in}}=0\right)$. The expression for the frequency-dependent reflection coefficient is given by

$$
r(\omega) \equiv \frac{V_{L}^{\text {out }}(\omega)}{V_{L}^{\text {in }}(\omega)}=\frac{C_{c} Z_{0} \omega\left(\frac{\omega^{2}}{\omega_{J}^{2}}-1\right)}{2 i\left(1-\frac{\omega^{2}}{\omega_{0}^{2}}\right)+C_{c} Z_{0} \omega\left(\frac{\omega^{2}}{\omega_{J}^{2}}-1\right)},
$$

where $\omega_{0}=1 / \sqrt{L_{J}\left(C_{c}+C_{J}\right)}$ is the resonance frequency of the coupled transmon and $\omega_{J}=1 / \sqrt{L_{J} C_{J}}$ is the resonance frequency of the bare (uncoupled) transmon. In Fig. 2 the reflection around the transmon resonance frequencies is shown for different values of $Z_{0}$. We see that for low impedance $Z_{0} C_{c} \omega<1$, the reflection is weak, except at $\omega_{0}$, where it is unity due to resonant reflection from the transmon [68]. For high impedance $Z_{0} C_{c} \omega>1$ we instead see strong reflection at all frequencies, except around the "new" resonance frequency $\omega_{J}$, where we find zero reflection independent of $Z_{0}$. The crossover occurs at $Z_{0} \sim Z_{J} C_{J} / C_{c}$, introducing the transmon impedance

$$
Z_{J}=\sqrt{\frac{L_{J}}{C_{J}}}=R_{Q} \sqrt{\frac{2 E_{C}}{E_{J}}},
$$

where $E_{C}=e^{2} /\left(2 C_{J}\right)$ is the charging energy of the transmon.

In the high-impedance regime, the strong scattering away from $\omega_{J}$ occurs due to the comparably strong capacitive 
coupling to the ground at the transmon without exciting the transmon. Close to $\omega_{J}$, the resonantly excited transmon counteracts this capacitive coupling and effectively acts like an open circuit. This is the opposite behavior compared to the low-impedance regime, where the transmon is effectively an open circuit at all frequencies, except at its resonance frequency $\omega_{0}$, where it acts like a shorted circuit, giving full reflection.

By expanding Eq. (5) around its pole close to $\omega_{J}$, we can extract the high-impedance coupling strength $\gamma_{J}=2 / Z_{0} C_{J}$, which, in contrast to the low-impedance expression $\gamma_{0}=$ $Z_{0} C_{c}^{2} / 2 L_{J}\left(C_{J}+C_{c}\right)^{2}$, does not depend on $C_{c}$ and decreases with increasing $Z_{0}$. In this regime, the voltage $V_{0}=V_{L}^{\text {in }}+$ $V_{L}^{\text {out }}=V_{R}^{\text {in }}+V_{R}^{\text {out }}$ at the node coupling to the TL oscillates with the full voltage across the JJ, $V_{J}=\dot{\phi}_{J}$. Due to the large $Z_{0}$, the amount of current passing through the TL is small $\left(\propto V_{J} / Z_{0}\right)$ and is thus not able to change the voltage $V_{0}-V_{J}$ across $C_{c}$ significantly, i.e., $\left|V_{0}-V_{J}\right| \ll V_{J}$. More details of the derivation can be found in Appendix B.

In the low-impedance regime, we instead have large currents flowing through the TL, keeping the voltage at the coupling node close to zero, i.e., $\left|V_{0}\right| \ll\left|V_{J}\right|$. These currents obviously scale with $C_{c}$, and the energy dissipation scales with $Z_{0}$, explaining the expression for $\gamma_{0}$. In the following, we investigate how the mirror affects the scattering.

\section{B. Mirror}

The mirror couples the fields to the right of the transmon $V_{R}^{\text {in }}(t)=-V_{R}^{\text {out }}(t-T)$, introducing the time of propagation to the mirror and back $T=2 L / v$ [see Fig 1(b)]. Like before, we can find the response to a harmonic field incoming from the left by Fourier transformation of the equations of motion. Since the absolute value of the reflection for the transmon in front of a mirror is always unity, we are now interested in the frequency dependence of the ratio between the trapped field (between the qubit and the mirror) and the incoming field, which is given by $f(\omega) \equiv V_{R}^{\text {out }}(\omega) / V_{L}^{\text {in }}(\omega)$,

$$
f(\omega)=\frac{\left(\frac{\omega^{2}}{\omega_{0}^{2}}-1\right)}{\left(1-\frac{\omega^{2}}{\omega_{0}^{2}}\right)-i \frac{C_{c} Z_{0} \omega}{2}\left(\frac{\omega^{2}}{\omega_{J}^{2}}-1\right)\left(e^{i \omega T}-1\right)},
$$

which is shown in Fig. 3. In the high-impedance regime, we now find cavity resonances between the highly reflective atom and the mirror when the frequency is close to $n \omega_{c}$ for $n=$ $1,2, \ldots$ and $\omega_{c}=2 \pi / T$, as shown by the peaks in the inset of Fig. 3. These are broadened by the coupling to the TL by $\gamma_{c}^{n}=\left|t\left(n \omega_{c}\right)\right|^{2} / T$, where $t(\omega)$ is the transmission across the transmon and $|t(\omega)|^{2}=1-|r(\omega)|^{2}$.

We find that the effect of the mirror on the transmon resonance close to $\omega_{J}$ is simply to reduce its broadening by a factor of 2 to $\gamma_{J}^{m}=1 / Z_{0} C_{J}$, away from any qubit-cavity resonance $\omega_{J} \approx n \omega_{c}$. As shown in the main panel of Fig. 3, on resonance $\omega_{J} \approx n \omega_{c}$ we find an avoided crossing with the coupling strength

$$
\Omega_{n}=\frac{2 \omega_{J}}{\sqrt{2 \pi n C_{J} Z_{0} \omega_{J}}}=\frac{2 \omega_{J}}{\sqrt{2 \pi n \frac{Z_{0}}{Z_{J}}}},
$$

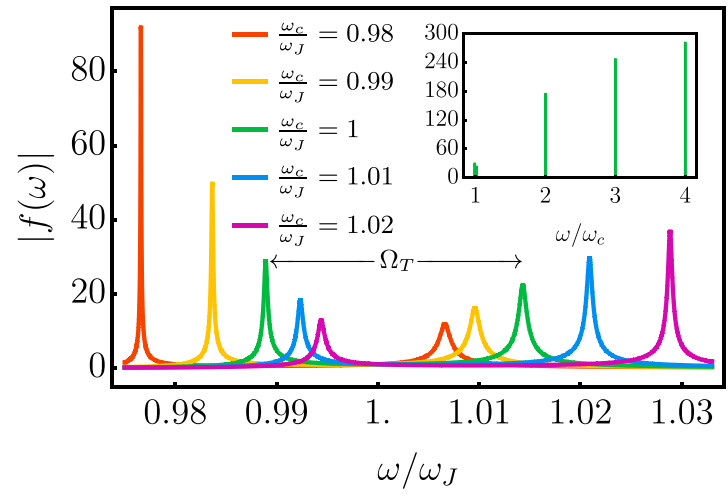

FIG. 3. Amplitude of the electromagnetic field between the qubit and the mirror $|f(\omega)|=\left|V_{R}^{\text {out }}(\omega) / V_{L}^{\text {in }}(\omega)\right|$ for $\frac{C_{c}}{C_{J}}=0.1$ and $Z_{0} / Z_{J}=$ 1000. The delay time is given by $T=2 \pi n / \omega_{c}, n=1$, and $\omega_{c}$ varies with respect to $\omega_{J}$. The different colors show $\omega_{c} / \omega_{J}=0.98$ (red), $\omega_{c} / \omega_{J}=0.99$ (yellow), $\omega_{c} / \omega_{J}=1$ (green), $\omega_{c} / \omega_{J}=1.01$ (blue), and $\omega_{c} / \omega_{J}=1.02$ (purple). All curves show a splitting which can be understood as a vacuum Rabi splitting. Without detuning between $\omega_{J}$ and $\omega_{c}$, the splitting is centered exactly around $\omega / \omega_{J}=1$. The inset shows the appearance of higher cavity modes appearing at $\omega \approx n \omega_{c}$, where $\omega_{c} T=2 \pi$ for $\omega_{c} / \omega_{J}=1$, corresponding to the green curve in the main plot.

which we derived by analyzing the splitting of the poles of the Laplace transform of the equations of motion in the highimpedance regime. More details can be found in Appendix A. Using the resonance condition $\omega_{J} \approx n \omega_{c}$ again, we note that the $n$ dependence cancels on resonance,

$$
\Omega_{T}=\frac{2}{\sqrt{T C_{J} Z_{0}}} .
$$

As we will see in the next section, where we investigate the spontaneous emission dynamics of the transmon, we find that this coupling indeed gives rise to vacuum Rabi oscillations between the transmon and the cavity mode.

In the low-impedance regime $Z_{0} C_{c} \omega<1, f(\omega)$ is instead close to unity, indicating only a little scattering from the transmon for all frequencies far from the transmon resonance $\omega=\omega_{0}$. Here, $f\left(\omega_{0}\right)=0$ since the field is reflected by the transmon and does not reach the mirror. When the transmon is located at a distance corresponding to a node of the electromagnetic field at its resonance frequency, i.e., $\omega_{0} T=2 n \pi$, it is in a dark state and is thus completely invisible to the incoming field at frequency $\omega_{0}$, giving instead $f\left(\omega_{0}\right)=1$. In the dark state, both the transmon and the field between the transmon and the mirror are excited. Thus, if the distance is slightly longer or shorter than the node, the state is no longer completely dark, and we instead get a pronounced scattering resonance $(|f(\omega)| \gg 1)$ at frequencies slightly lower or higher than $\omega_{0}$ (see, e.g., the red line in Fig. 3). For higher $Z_{0}$, we see that this dark state resonance moves in frequency towards the cavity frequency $\omega_{c}=2 \pi / T$. As shown in Appendix A, for small $C_{c} / C_{J} \ll 1$ and $\omega_{0}=n \omega_{c}$, we can find vacuum Rabi oscillations damped towards a finite dark state population.

In the following, we investigate how the high-impedance TL influences spontaneous emission of the transmon. 


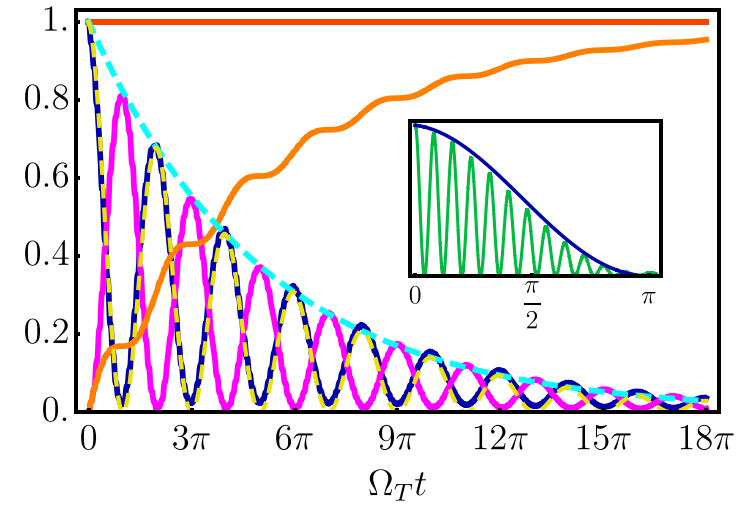

FIG. 4. Energy of the transmon (blue), field between the mirror and transmon (pink), outgoing field to the left (orange), sum of all energies (red), approximated decay $\gamma_{J}^{m}$ (dashed cyan), and approximated Rabi frequency $\Omega_{T}$ and decay $e^{-\gamma_{J}^{m} t / 2} \cos ^{2}\left(\Omega_{T} t / 2\right)$ (dashed yellow) for $C_{c} / C_{J}=0.5, Z_{0} / Z_{J}=100, T=2 \pi / \omega_{c}, n \omega_{c}=\omega_{J}, n=$ 1 as a function of periods of the Rabi oscillations. The inset shows the energy of the transmon (blue) and the flux $\phi_{J}^{2}$ (green) for the first oscillation of the frequency $\Omega_{T}$. This clearly shows the different timescales of the energy compared to the variables of the transmon.

\section{SPONTANEOUS EMISSION AND RABI OSCILLATIONS}

We consider the case of a transmon initially excited at time $t=0$ with a finite flux $\phi_{J}(0)>0$, while the other qubit variables are zero $p_{J}(0)=p_{0}(0)=0$ and the TL is in the vacuum state. The qubit energy

$$
E_{q}(t)=\frac{\left[p_{J}(t)+p_{0}(t)\right]^{2}}{2 C_{J}}+\frac{p_{0}(t)^{2}}{2 C_{c}}+\frac{\phi_{J}(t)^{2}}{2 L_{J}},
$$

is the sum of the capacitive energy on the two capacitances and the inductive energy in the JJ. The current amplitude emitted from the transmon into the TL is $\partial_{t} p_{0}(t)$, and from this we can write the change in the energy $E_{R}(t)$ of the field between the transmon and the mirror as

$$
\partial_{t} E_{R}(t)=\frac{Z_{0}}{4}\left\{\left[\partial_{t} p_{0}(t)\right]^{2}-\left[\partial_{t} p_{0}(t-T)\right]^{2}\right\},
$$

where the first term corresponds to the instantaneous power emitted into the TL and the second term is the instantaneous power coming back from the mirror. The change in the energy of the field to the left of the transmon $E_{L}(t)$ is given by the instantaneous left-moving power leaving the system,

$$
\partial_{t} E_{L}(t)=\frac{Z_{0}}{4}\left[\partial_{t} p_{0}(t)-\partial_{t} p_{0}(t-T)\right]^{2},
$$

where the left-moving current amplitude is a sum of the current emitted by the transmon and the delayed current arriving from the mirror.

In Fig. 4, we plot these energies for $Z_{0} / Z_{J}=100$ for the case of resonance between the transmon and the first cavity resonance $\omega_{J}=\omega_{c}$. The system energies indeed perform damped Rabi oscillations with the frequency $\Omega_{T}=$ $2 / \sqrt{T C_{J} Z_{0}}$ and half the off-resonance damping rate $\gamma_{J}^{m} / 2=$ $1 / 2 C_{J} Z 0$, as indicated by the yellow line given by the expression $e^{-\gamma_{J}^{m} t / 2} \cos ^{2}\left(\Omega_{T} t / 2\right)$, approximating the full numerical solution of the differential equations very well. We note that Laplace transforming the equations of motion (1)-(4) and

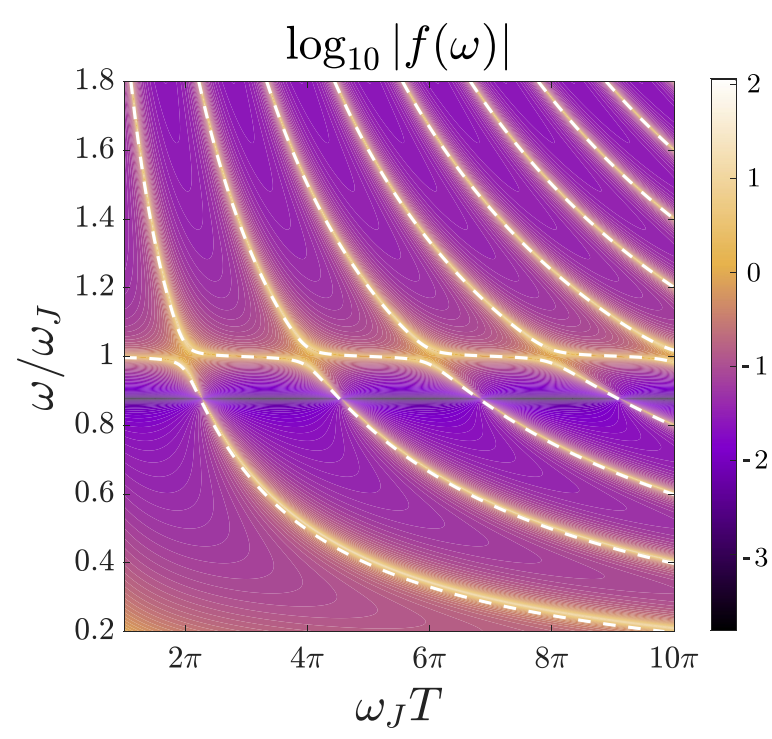

FIG. 5. Response function (log color scale) versus normalized frequency $\omega / \omega_{J}$ and linearly varied time delay $\omega_{J} T$. The eigenvalues $\Omega_{\alpha}$ are computed for $N=8$ cavity modes and are shown as superimposed white dashed lines. The parameters are chosen to be $Z_{0} / Z_{J}=100$ and $C_{c} / C_{J}=0.3$.

calculating the residues of the system variables give expressions for the Rabi frequency and damping rate similar to the analysis of the resonances in the scattering amplitudes. More details and a comparison of the approximation to the numerical results are found in Appendix A.

\section{EFFECTIVE QUANTUM MODEL: ATOM IN A MULTIMODE CAVITY HAMILTONIAN}

We now go on to demonstrate that in the high-impedance regime the response function $f(\omega)$ of the field trapped between the transmon and the mirror (7) reproduces the dynamics of an effective Hamiltonian of a single transition atom in a multimode cavity. This Hamiltonian is of the form

$$
\begin{aligned}
H= & \omega_{J}\left(a^{\dagger} a+\frac{1}{2}\right)+\sum_{n=1}^{\infty} n \omega_{c}\left(c_{n}^{\dagger} c_{n}+\frac{1}{2}\right) \\
& +\sum_{n=1}^{\infty} \frac{\Omega_{n}}{2}\left(a^{\dagger}+a\right)\left(c_{n}^{\dagger}+c_{n}\right),
\end{aligned}
$$

where the operators $a, a^{\dagger}$ are annihilation and creation bosonic operators $\left(\left[a, a^{\dagger}\right]=1\right)$ associated with excitation in the transmon qubit, while $c_{n}, c_{n}^{\dagger}$ annihilate and create photons in the cavity modes. When weakly excited, the choice of bosonic excitations of the transmon is justified, while the orthogonality relations between the cavity modes is ensured by the high finesse of the latter, so that we have $\left[c_{n}, c_{m}^{\dagger}\right]=\delta_{n m}$. Details of the diagonalization of the Hamiltonian are shown in Appendix $C$. The response function $|f(\omega)|$ and eigenfrequencies of Hamiltonian (13) are shown in Fig. 5. The eigenfrequencies are shown to match the peaks of $|f(\omega)|$ for all cavity modes. Noticeably, a dip in the response function corresponding to the dark state is found for $\omega=\omega_{0}$. A similar multimode 

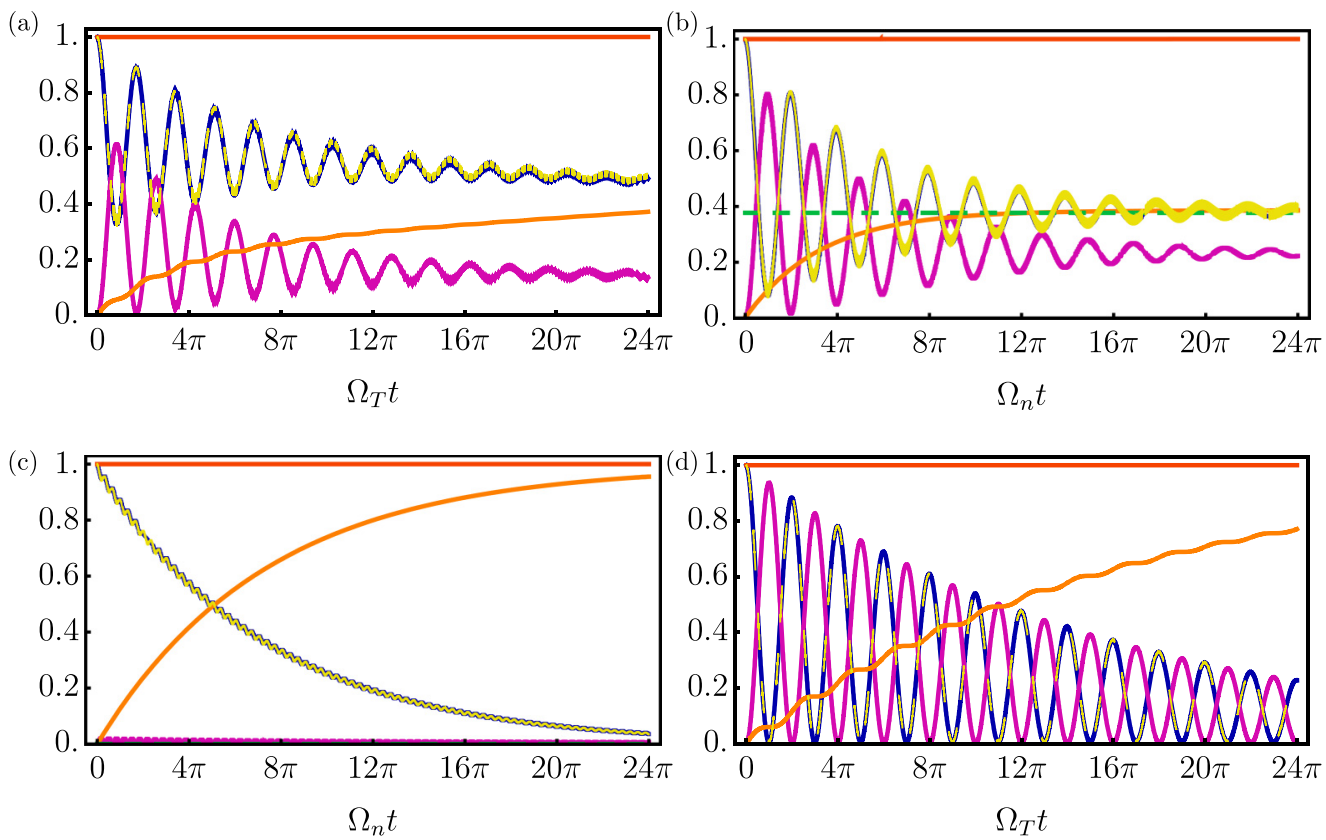

FIG. 6. Energy of the transmon (blue), energy of the field trapped between the transmon and the mirror (magenta), outgoing field to the left side of the transmon (orange), total energy of the system (red), and semi-numerically calculated energy of the transmon using Eq. (A2) (yellow dashed) as a function of the period of the Rabi oscillations. The parameters for the panels are the following: (a) $Z_{0} / Z_{J}=1000, C_{c} / C_{J}=0.02$, $T=2 \pi / \omega_{c}$, and $\omega_{J}=n \omega_{c}, n=1$. Since we chose $C_{c} / C_{J}=0.02$, the decay is very slow. (b) $Z_{0} / Z_{J}=1000, C_{c} /\left(C_{J}+C_{c}\right)=0.02, T=2 \pi / \omega_{c}$, and $\omega_{0}=n \omega_{c}, n=1$. Here, the parameters are almost the same as in (a), but now the dark state condition $\omega_{c}=\omega_{0}$ is fulfilled. The system converges into a dark state with a finite-excitation probability of both the transmon and the field between the transmon and the mirror. The green line indicates the analytical value of the dark state energy $\frac{E_{D S}}{E_{0}}=\frac{1}{\left(1+\frac{T}{2} \gamma_{0}\right)^{2}}$, with $\gamma_{0}=\frac{Z_{0} \omega_{0}^{2}}{2} \frac{C_{c}^{2}}{C_{c}+C_{J}}$. (c) $Z_{0} / Z_{J}=1000, C_{c} /\left(C_{J}+C_{c}\right)=0.3$, $T=2 \pi / \omega_{c}$ and $\omega_{0}=n \omega_{c}, n=1$. Here, although $\omega_{0}=\omega_{C}$ as in (b), the system does not seem to converge into a dark state, and the decay rate is given by $\gamma_{J}=1 / C_{J} Z_{0}$. The difference from (b) is that the ratio between $\frac{C_{c}}{C_{J}} \frac{Z_{0}}{Z_{J}}$ here is much bigger than in (b), which also means that $\omega_{J}$ is not close to $\omega_{C}$ and the Rabi oscillations are barely visible. (d) $Z_{0} / Z_{J}=1000, C_{c} / C_{J}=0.3, T=2 \pi \omega_{c}$, and $\omega_{J}=n \omega_{c}$, $n=1$. Here, as in (c) $\frac{C_{c}}{C_{J}} \frac{Z_{0}}{Z_{J}} \gg 1$, but the "Rabi condition" $\omega_{J}=n \omega_{C}$ is still fulfilled. We see clear Rabi oscillations, the decay is slower compared to (c), and the decay rate is given by $\gamma_{J}^{m} / 2=1 / 2 C_{J} Z_{0}$.

Hamiltonian with a $(1 / \sqrt{n})$-dependent coupling was studied in Refs. [69,70].

\section{DISCUSSION AND OUTLOOK}

We have theoretically investigated the properties of a transmon capacitively coupled to a high-impedance transmission line, a system which is currently becoming experimentally accessible. By linearizing the Josephson junction, we could describe the low-excitation dynamics, including spontaneous emission. We find qualitatively different behavior compared to that of the low-impedance regime. In particular, the atom now forms its own cavity, and we can observe a vacuum Rabi splitting, giving rise to Rabi oscillations in the spontaneous emission. The system is well described by a Hamiltonian for an atom weakly coupled to a multimode cavity. We hope that this analysis will inspire an experimental realization of this novel system.

\section{ACKNOWLEDGMENTS}

The authors thank A. Parra-Rodriguez for important comments on the manuscript. The authors acknowledge funding from the Swedish Research Council (VR) through Grant No. 2016-06059. G.J. also acknowledges funding from the Knut and Alice Wallenberg Foundation (KAW) through the Wallenberg Centre for Quantum Technology (WACQT).

\section{APPENDIX A: RABI OSCILLATIONS OF AN ATOM COUPLED TO A HIGH-IMPEDANCE SEMI-INFINITE TL: ADDITIONAL PARAMETER VALUES}

We can Laplace transform the equations of motion of the transmon shown in the main text and extract the exact poles numerically. With the following formulas we can calculate the inverse Laplace transform of the system variables and energy:

$$
\begin{gathered}
\operatorname{Res}_{1,2}^{ \pm} p_{J}(s)=\lim _{s \rightarrow s_{1,2}^{ \pm}} p_{J}(s)\left(s-s_{1,2}^{ \pm}\right), \\
p_{J}(t)=\int p_{J}(s) e^{-s t} d s=\left.2 \pi i \sum_{k} \operatorname{Res} p_{J}(s) e^{-s t}\right|_{s=s_{k}},
\end{gathered}
$$

where $k=s_{1,2}^{ \pm}$are the poles of $p_{J}(s)$. Similarly, we calculate $\phi_{J}(t)$. We show the results as an addition to Fig. 4 in the main text. Here, we provide more figures for different system parameters. In all panels of Fig. 6, the impedance is chosen to be $Z_{0} / Z_{J}=1000$. In Fig. $6(\mathrm{a})$, the ratio of the coupling capacitance and the Josephson capacitance is fairly small, $C_{c} / C_{J}=0.02$, and the coupling to the TL is weak. The cavity frequency equals the resonance frequency of the 


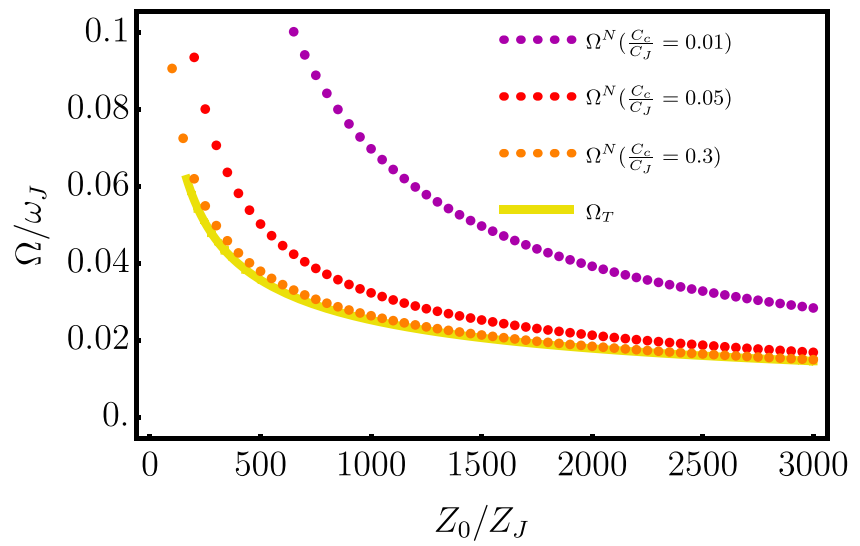

FIG. 7. Rabi frequency $\Omega$ as a function of $Z_{0} / Z_{J}$ calculated analytically $\Omega_{T}$ (solid yellow line) and numerically $\Omega^{N}$ (dots) for different values of $C_{c} / C_{J}, C_{c} / C_{J}=0.01$ (purple), $C_{c} / C_{J}=0.05$ (red), $C_{c} / C_{J}=0.3$ (orange), for $T=2 \pi / \omega_{c}, \omega_{J}=n \omega_{c}, n=1$. We see that the higher $\frac{C_{c}}{C_{J}} \frac{Z_{0}}{Z_{J}}$ becomes, the closer the approximated analytical frequency $\Omega_{T}$ and the numerically calculated frequency $\Omega^{N}$ become.

transmon $\omega_{c}=\omega_{J}$. The decay is weak, and the Rabi oscillations are clearly visible. The parameters in Fig. 6(b) are similar to those of Fig. 6(a), but now $C_{c} /\left(C_{J}+C_{c}\right)=0.02$, and most importantly, the cavity frequency equals the transition frequency of the qubit for low impedance, $\omega_{c}=\omega_{0}$, which is the condition for a dark state [61]. The energy of the transmon decays until it reaches the dark state, with energy $\frac{E_{D S}}{E_{0}}=\frac{1}{\left(1+\frac{T}{2} \gamma_{0}\right)^{2}}$, where $\gamma_{0}=\frac{Z_{0} \omega_{0}^{2}}{2} \frac{C_{c}^{2}}{C_{c}+C_{J}}$ [61]. In Fig. 6(c), the coupling capacitance is much larger than those of Figs. 6(a) and 6(b), $C_{c} /\left(C_{c}+C_{J}\right)=0.3$, and the transmon fulfills the dark state condition $\omega_{c}=\omega_{0}$. Anyhow, the system does not converge into a dark state, and the Rabi oscillations are very weak. The main difference here is that, since $C_{c} /\left(C_{c}+C_{J}\right)$ is rather large, $\omega_{J}$ is not close to $\omega_{c}$, and the Rabi oscillations and coupling to the cavity are suppressed. In this parameter regime, the behavior of the system seems to be independent of the position of the transmon with respect to the mirror. Similar to Fig. 6(c), in Fig. 6(d), the coupling capacitance is rather large too, $C_{c} / C_{J}=0.3$, but here, the cavity frequency equals the frequency of the transmon $\omega_{c}=\omega_{J}$, which means that the Rabi condition is fulfilled. We see clear Rabi oscillations, and in addition, the decay is much slower than in Fig. 6(c).

As mentioned in the main text, we find an analytical expression for the oscillation frequency by analyzing the Laplace transform of the equations of motion. The denominator of all system variables in Laplace transform is given by

$$
N(s)=2 e^{s T}\left(1+\frac{s^{2}}{\omega_{0}^{2}}\right)+s\left(1-e^{s T}\right)\left(1+\frac{s^{2}}{\omega_{J}^{2}}\right) C_{c} Z_{0} .
$$

If we expand the denominator $N(s)$ around the resonance frequency $s \rightarrow \omega_{J}(i+x+i y)$, with $x, y \ll 1$ for high $\frac{C_{c}}{C_{J}} \frac{Z_{0}}{Z_{J}}$, we find that the splitting of the poles, and hence the Rabi frequency, is given by $\Omega_{n}=\frac{2 \omega_{J}}{\sqrt{2 \pi n C_{J} Z_{0} \omega_{J}}}$. In Fig. 7, we demonstrate the deviations of the approximation from numerically calculated values. We find that the higher the ratio $\frac{C_{c}}{C_{I}} \frac{Z_{0}}{Z_{J}}$ is, the more closely the approximation resembles the numerical solution.

\section{APPENDIX B: ANALYSIS OF THE RESPONSE FUNCTIONS}

To analyze the solution of the Fourier transformation of the equations of motion in the main text in a convenient manner, we introduce the following functions:

$$
\begin{gathered}
N(\omega)=R_{0}(\omega)-i R_{J}(\omega), \\
R_{0}(\omega)=\left(1-\frac{\omega^{2}}{\omega_{0}^{2}}\right) \\
R_{J}(\omega)=\frac{C_{c} Z_{0}}{2} \omega\left(1-\frac{\omega^{2}}{\omega_{J}^{2}}\right) .
\end{gathered}
$$

With these definitions we are able to write the transmission and the reflection amplitudes of the qubit in the open transmission line as

$$
r(\omega)=\frac{i R_{J}(\omega)}{N(\omega)}, \quad t(\omega)=\frac{R_{0}(\omega)}{N(\omega)}, \quad r(\omega)+1=t(\omega) .
$$

We note that the high-impedance regime corresponds to $\left|R_{J}(\omega)\right| \gg\left|R_{0}(\omega)\right|$ away from resonances, i.e., $\left|C_{c} Z_{0} \omega / 2\right| \gg$ 1 , while the opposite $\left(\left|R_{J}(\omega)\right| \ll\left|R_{0}(\omega)\right|\right)$ is true in the lowimpedance regime, $\left|C_{c} Z_{0} \omega / 2\right| \ll 1$.

\section{Damping rate for the open TL}

We analyze the scattering solution of the qubit excitation $\phi_{J}$ to find the damping rate for the transmon in an open TL. The solution in frequency space reads

$$
\phi_{J}(\omega)=-\frac{C_{c} L_{J} \omega}{R_{0}(\omega)-R_{J}(\omega)} .
$$

In the high-impedance regime, we perform an expansion around the bare qubit frequency $\omega=\omega_{J}+\delta \omega$ and find, to first order in $\delta \omega$,

$$
\phi_{J}\left(\omega_{J}+\delta \omega\right) \approx \frac{i}{\omega_{J}} \frac{1}{1-i 2 \delta \omega / \gamma_{J}},
$$

where $\gamma_{J}=2 / Z_{0} C_{J}$ is the energy damping rate for spontaneous emission.

In the low-impedance regime, the qubit resonance is shifted to $\omega_{0}$, and we instead expand $\omega=\omega_{0}+\delta \omega$ to find

$$
\phi_{J}\left(\omega_{0}+\delta \omega\right)=\frac{1}{C_{c} Z_{0} \omega_{0}^{2}} \frac{1}{1-i 2 \delta \omega / \gamma_{0}},
$$

where $\gamma_{0}=\frac{Z_{0} \omega_{0}^{2}}{2} \frac{C_{c}^{2}}{C_{c}+C_{J}}$ is the low-impedance damping rate [61].

\section{Damping rates and Lamb shifts with a mirror}

With a mirror, the solution of the Josephson flux $\phi_{J}$ can be written as

$$
\phi_{J}(\omega)=\frac{-i C_{c} L_{J} \omega\left(1-e^{i \omega T}\right)}{R_{0}(\omega)-i R_{J}(\omega)\left(1-e^{i \omega T}\right)} .
$$

In the low-impedance regime, we find that the qubit resonance is Lamb shifted to $\tilde{\omega}_{0}=\omega_{0}+\gamma_{0} \sin \left(\omega_{0} T\right) / 2$, and we can 
expand $\phi_{J}(\omega)$ around the resonance and find

$$
\phi_{J}\left(\tilde{\omega}_{0}+\delta \omega\right) \approx-i \frac{C_{c}}{C_{J}+C_{c}} \frac{e^{i \omega_{0} T / 2}}{\gamma_{0} \sin \left(\omega_{0} T / 2\right)} \frac{1}{1-i 2 \delta \omega / \gamma_{0}^{m}},
$$

with the Purcell-modified damping rate $\gamma_{0}^{m}=$ $2 \gamma_{0} \sin ^{2}\left(\omega_{0} T / 2\right)$.

In the high-impedance regime, the resonance frequency is Lamb shifted to $\tilde{\omega}_{J}=\omega_{J}+\gamma_{J} \cot \left(\omega_{J} T / 2\right) / 4$. Expanding $\phi_{J}(\omega)$ around this frequency, we find

$$
\phi_{J}\left(\tilde{\omega}_{J}+\delta \omega\right)=-\frac{2 i}{\omega_{J}} \frac{1}{1+i 2 \delta \omega / \gamma_{J}^{m}},
$$

where $\gamma_{J}^{m}=\gamma_{J} / 2=1 / C_{J} Z_{0}$ is the damping rate of an atom in front of a mirror in the high- $Z_{0}$ regime. Here, we note that the expression for the Lamb shift diverges when $\sin \left(\omega_{J} T / 2\right)=0$, i.e., when $\omega_{J}$ is close to a cavity resonance $\omega_{c}^{n}=2 \pi n / T$. This is when the single pole approximation is no longer valid and we find the vacuum Rabi splitting. Away from the Rabi condition, we also note that the damping rate is independent of the distance to the mirror; that is, we see no Purcell effect. Away from the Rabi condition, we can also analyze the response function

$$
f(\omega)=\frac{1}{1-i \frac{R_{J}(\omega)}{R_{0}(\omega)}\left(1-e^{i \omega T}\right)}
$$

to extract the cavity modes. Due to the finite transmission through the transmon, they are slightly shifted from the perfect mirror frequencies to $\tilde{\omega}_{c}^{n}=\omega_{c}^{n}+R_{0}\left(\omega_{c}^{n}\right) / T R_{J}\left(\omega_{c}^{n}\right)$. Close to the resonances we can expand $\omega=\tilde{\omega}_{c}^{n}+\delta \omega$ and find

$$
f\left(\tilde{\omega}_{c}^{n}+\delta \omega\right) \approx \frac{2}{t\left(\omega_{c}^{n}\right)} \frac{1}{1-i 2 \delta \omega / \gamma_{c}^{n}},
$$

where $\gamma_{c}^{n} \approx\left|t\left(\tilde{\omega}_{c}^{n}\right)\right|^{2} / T$ is the energy damping rate of cavity mode $n$.

\section{APPENDIX C: HOPFIELD DIAGONALIZATION OF THE ATOM IN A MULTIMODE CAVITY HAMILTONIAN}

To diagonalize the effective quantum optical Hamiltonian [Eq. (13) in the main text], we first introduce the new polaritonic operators

$$
\Pi_{\alpha}=x^{\alpha} a+y^{\alpha} a^{\dagger}+\sum_{n}\left(m_{n}^{\alpha} c_{n}+h_{n}^{\alpha} c_{n}^{\dagger}\right),
$$

with $x^{\alpha}, y^{\alpha}, m_{n}^{\alpha}, h_{n}^{\alpha}$ being the Hopfield coefficients associated with each bosonic operator $a, a^{\dagger}, c_{n}, c_{n}^{\dagger}$. To ensure the bosonicity of the polaritonic operators $\left(\left[\Pi_{\alpha}, \Pi_{\beta}^{\dagger}\right]=\delta_{\alpha \beta}\right)$, the coefficients should satisfy the relation

$$
\left|x^{\alpha}\right|^{2}-\left|y^{\alpha}\right|^{2}+\sum_{n}\left(\left|m_{n}^{\alpha}\right|^{2}-\left|h_{n}^{\alpha}\right|^{2}\right)=1 .
$$

The new operators should satisfy the eigenvalue problem

$$
\left[\Pi_{\alpha}, H\right]=\Omega_{\alpha} \Pi_{\alpha},
$$

where $\Omega_{\alpha}$ are the eigenfrequencies labeled with a new index $\alpha$. Typically, if we couple the atom with $N$ cavity modes, then $\alpha$ runs from 1 to $N+1$. Expanding the commutator in the previous equation, it is possible to write the eigenvalue problem in matrix form:

$$
\left(\begin{array}{ccccccc}
\omega_{J} & 0 & g_{1} & -g_{1} & \ldots & g_{N} & -g_{N} \\
0 & -\omega_{J} & g_{1} & -g_{1} & \ldots & g_{N} & -g_{N} \\
g_{1} & -g_{1} & \omega_{1} & 0 & \ldots & 0 & 0 \\
g_{1} & -g_{1} & 0 & -\omega_{1} & & 0 & 0 \\
\vdots & \vdots & \vdots & & \ddots & & \vdots \\
g_{N} & -g_{N} & 0 & 0 & & \omega_{N} & 0 \\
g_{N} & -g_{N} & 0 & 0 & \ldots & 0 & -\omega_{N}
\end{array}\right)\left(\begin{array}{c}
x^{\alpha} \\
y^{\alpha} \\
m_{1}^{\alpha} \\
h_{1}^{\alpha} \\
\vdots \\
m_{N}^{\alpha} \\
h_{N}^{\alpha}
\end{array}\right)=\Omega_{\alpha}\left(\begin{array}{c}
x^{\alpha} \\
y^{\alpha} \\
m_{1}^{\alpha} \\
h_{1}^{\alpha} \\
\vdots \\
m_{N}^{\alpha} \\
h_{N}^{\alpha}
\end{array}\right) .
$$

This eigenvalue problem can be solved analytically for $N=1$, but in general, one has to diagonalize it numerically. A comparison between the full response function and the eigenvalues is shown in Fig. 5 in the main text.

[1] D. Roy, C. M. Wilson, and O. Firstenberg, Colloquium: Strongly interacting photons in one-dimensional continuum, Rev. Mod. Phys. 89, 021001 (2017).

[2] X. Gu, A. F. Kockum, A. Miranowicz, Y.-X. Liu, and F. Nori, Microwave photonics with superconducting quantum circuits, Phys. Rep. 718-719, 1 (2017).

[3] A. González-Tudela, V. Paulisch, H. J. Kimble, and J. I. Cirac, Efficient Multiphoton Generation in Waveguide Quantum Electrodynamics, Phys. Rev. Lett. 118, 213601 (2017).

[4] V. Paulisch, H. J. Kimble, J. I. Cirac, and A. González-Tudela, Generation of single- and two-mode multiphoton states in waveguide QED, Phys. Rev. A 97, 053831 (2018).
[5] F. Quijandría, I. Strandberg, and G. Johansson, Steady-State Generation of Wigner-Negative States in One-Dimensional Resonance Fluorescence, Phys. Rev. Lett. 121, 263603 (2018).

[6] A. Wallraff, D. I. Schuster, A. Blais, L. Frunzio, R.-S. Huang, J. Majer, S. Kumar, S. M. Girvin, and R. J. Schoelkopf, Strong coupling of a single photon to a superconducting qubit using circuit quantum electrodynamics, Nature (London) 431, 162 (2004).

[7] A. Blais, R.-S. Huang, A. Wallraff, S. M. Girvin, and R. J. Schoelkopf, Cavity quantum electrodynamics for superconducting electrical circuits: An architecture for quantum computation, Phys. Rev. A 69, 062320 (2004). 
[8] G. Wendin, Quantum information processing with superconducting circuits: A review, Rep. Prog. Phys. 80, 106001 (2017).

[9] A. F. Kockum and F. Nori, Quantum bits with Josephson junctions, in Fundamentals and Frontiers of the Josephson Effect, edited by F. Tafuri (Springer, Cham, 2019), pp. 703-741.

[10] B. Kannan, M. J. Ruckriegel, D. L. Campbell, A. Frisk Kockum, J. Braumüller, D. K. Kim, M. Kjaergaard, P. Krantz, A. Melville, B. M. Niedzielski, A. Vepsäläinen, R. Winik, J. L. Yoder, F. Nori, T. P. Orlando, S. Gustavsson, and W. D. Oliver, Waveguide quantum electrodynamics with superconducting artificial giant atoms, Nature (London) 583, 775 (2020).

[11] A. Frisk Kockum, Quantum optics with giant atoms-The first five years, in International Symposium on Mathematics, Quantum Theory, and Cryptography, edited by T. Takagi, M. Wakayama, K. Tanaka, N. Kunihiro, K. Kimoto, and Y. Ikematsu (Springer, Singapore, 2021), pp. 125-146.

[12] H. Zheng, D. J. Gauthier, and H. U. Baranger, Waveguide QED: Many-body bound-state effects in coherent and Fock-state scattering from a two-level system, Phys. Rev. A 82, 063816 (2010).

[13] T. Tufarelli, F. Ciccarello, and M. S. Kim, Dynamics of spontaneous emission in a single-end photonic waveguide, Phys. Rev. A 87, 013820 (2013).

[14] T. Tufarelli, M. S. Kim, and F. Ciccarello, Non-Markovianity of a quantum emitter in front of a mirror, Phys. Rev. A 90, 012113 (2014).

[15] E. Sanchez-Burillo, D. Zueco, J. J. Garcia-Ripoll, and L. Martin-Moreno, Scattering in the Ultrastrong Regime: Nonlinear Optics with One Photon, Phys. Rev. Lett. 113, 263604 (2014).

[16] E. Sánchez-Burillo, L. Martín-Moreno, J. J. García-Ripoll, and D. Zueco, Full two-photon down-conversion of a single photon, Phys. Rev. A 94, 053814 (2016).

[17] E. Sánchez-Burillo, D. Zueco, L. Martín-Moreno, and J. J. García-Ripoll, Dynamical signatures of bound states in waveguide QED, Phys. Rev. A 96, 023831 (2017).

[18] M. Devoret, S. Girvin, and R. Schoelkopf, Circuit-QED: How strong can the coupling between a Josephson junction atom and a transmission line resonator be?, Ann. Phys. (Berlin, Ger.) 16, 767 (2007).

[19] M. Bamba and T. Ogawa, Recipe for the hamiltonian of systemenvironment coupling applicable to the ultrastrong-light-matterinteraction regime, Phys. Rev. A 89, 023817 (2014).

[20] A. Frisk Kockum, A. Miranowicz, S. De Liberato, S. Savasta, and F. Nori, Ultrastrong coupling between light and matter, Nat. Rev. Phys. 1, 19 (2019)

[21] D. Zueco and J. García-Ripoll, Ultrastrongly dissipative quantum Rabi model, Phys. Rev. A 99, 013807 (2019).

[22] J. A. Mlynek, A. A. Abdumalikov, C. Eichler, and A. Wallraff, Observation of Dicke superradiance for two artificial atoms in a cavity with high decay rate, Nat. Commun. 5, 5186 (2014).

[23] D. M. Pozar, Microwave Engineering (Wiley, Hoboken, NJ, 2005).

[24] J. Clarke and F. K. Wilhelm, Superconducting quantum bits, Nature, 453, 1031 (2008).

[25] I. V. Pechenezhskiy, R. A. Mencia, L. B. Nguyen, Y.-H. Lin, and V. E. Manucharyan, The superconducting quasicharge qubit, Nature 585, 368 (2020).

[26] D. Niepce, J. Burnett, and J. Bylander, High kinetic inductance NbN nanowire superinductors, Phys. Rev. Appl. 11, 044014 (2019).
[27] N. A. Masluk, I. M. Pop, A. Kamal, Z. K. Minev, and M. H. Devoret, Microwave Characterization of Josephson Junction Arrays: Implementing a Low Loss Superinductance, Phys. Rev. Lett. 109, 137002 (2012).

[28] T. Weißl, B. Küng, E. Dumur, A. K. Feofanov, I. Matei, C. Naud, O. Buisson, F. W. J. Hekking, and W. Guichard, Kerr coefficients of plasma resonances in Josephson junction chains, Phys. Rev. B 92, 104508 (2015).

[29] N. Samkharadze, A. Bruno, P. Scarlino, G. Zheng, D. P. DiVincenzo, L. DiCarlo, and L. M. K. Vandersypen, Highkinetic-inductance superconducting nanowire resonators for circuit QED in a magnetic field, Phys. Rev. Appl. 5, 044004 (2016).

[30] R. Kuzmin, R. Mencia, N. Grabon, N. Mehta, Y. H. Lin, and V. E. Manucharyan, Quantum electrodynamics of a superconductor-insulator phase transition, Nat. Phys. 15, 930 (2019).

[31] J. Puertas Martínez, S. Léger, N. Gheeraert, R. Dassonneville, L. Planat, F. Foroughi, Y. Krupko, O. Buisson, C. Naud, W. Hasch-Guichard, S. Florens, I. Snyman, and N. Roch, A tunable Josephson platform to explore many-body quantum optics in circuit-QED, npj Quantum Inf. 5, 19 (2019).

[32] M. T. Bell, I. A. Sadovskyy, L. B. Ioffe, A. Y. Kitaev, and M. E. Gershenson, Quantum Superinductor with Tunable Nonlinearity, Phys. Rev. Lett. 109, 137003 (2012)

[33] P. Winkel, I. Takmakov, D. Rieger, L. Planat, W. HaschGuichard, L. Grünhaupt, N. Maleeva, F. Foroughi, F. Henriques, K. Borisov, J. Ferrero, A. V. Ustinov, W. Wernsdorfer, N. Roch, and I. M. Pop, Nondegenerate parametric amplifiers based on dispersion-engineered Josephson-junction arrays, Phys. Rev. Appl. 13, 024015 (2020).

[34] Y. Krupko, V. D. Nguyen, T. Weiß1, E. Dumur, J. Puertas, R. Dassonneville, C. Naud, F. W. J. Hekking, D. M. Basko, O. Buisson, N. Roch, and W. Hasch-Guichard, Kerr nonlinearity in a superconducting Josephson metamaterial, Phys. Rev. B 98 094516 (2018).

[35] J. T. Peltonen, P. C. J. J. Coumou, Z. H. Peng, T. M. Klapwijk, J. S. Tsai, and O. V. Astafiev, Hybrid rf squid qubit based on high kinetic inductance, Sci. Rep. 8, 10033 (2018).

[36] L. Grünhaupt, N. Maleeva, S. T. Skacel, M. Calvo, F. LevyBertrand, A. V. Ustinov, H. Rotzinger, A. Monfardini, G Catelani, and I. M. Pop, Loss Mechanisms and Quasiparticle Dynamics in Superconducting Microwave Resonators Made of Thin-Film Granular Aluminum, Phys. Rev. Lett. 121, 117001 (2018).

[37] D. Niepce, J. J. Burnett, M. G. Latorre, and J. Bylander, Geometric scaling of two-level-system loss in superconducting resonators, Supercond. Sci. Technol. 33, 025013 (2020).

[38] V. E. Manucharyan, J. Koch, L. I. Glazman, and M. H. Devoret, Fluxonium: Single Cooper-pair circuit free of charge offsets, Science 326, 113 (2009).

[39] L. Grünhaupt, M. Spiecker, D. Gusenkova, N. Maleeva, S. T. Skacel, I. Takmakov, F. Valenti, P. Winkel, H. Rotzinger, W. Wernsdorfer, A. V. Ustinov, and I. M. Pop, Granular aluminium as a superconducting material for high-impedance quantum circuits, Nat. Mater. 18, 816 (2019).

[40] L. B. Nguyen, Y.-H. Lin, A. Somoroff, R. Mencia, N. Grabon, and V. E. Manucharyan, High-Coherence Fluxonium Qubit, Phys. Rev. X 9, 041041 (2019). 
[41] J. Koch, V. Manucharyan, M. H. Devoret, and L. I. Glazman, Charging Effects in the Inductively Shunted Josephson Junction, Phys. Rev. Lett. 103, 217004 (2009).

[42] I. M. Pop, K. Geerlings, G. Catelani, R. J. Schoelkopf, L. I. Glazman, and M. H. Devoret, Coherent suppression of electromagnetic dissipation due to superconducting quasiparticles, Nature (London) 508, 369 (2014).

[43] W. Guichard and F. W. J. Hekking, Phase-charge duality in Josephson junction circuits: Role of inertia and effect of microwave irradiation, Phys. Rev. B 81, 064508 (2010).

[44] A. Stockklauser, P. Scarlino, J. V. Koski, S. Gasparinetti, C. K. Andersen, C. Reichl, W. Wegscheider, T. Ihn, K. Ensslin, and A. Wallraff, Strong Coupling Cavity QED with Gate-Defined Double Quantum Dots Enabled by a High Impedance Resonator, Phys. Rev. X 7, 011030 (2017).

[45] J. Koch, T. M. Yu, J. Gambetta, A. A. Houck, D. I. Schuster, J. Majer, A. Blais, M. H. Devoret, S. M. Girvin, and R. J. Schoelkopf, Charge-insensitive qubit design derived from the Cooper pair box, Phys. Rev. A 76, 042319 (2007).

[46] I. C. Hoi, A. F. Kockum, L. Tornberg, A. Pourkabirian, G. Johansson, P. Delsing, and C. M. Wilson, Probing the quantum vacuum with an artificial atom in front of a mirror, Nat. Phys. 11, 1045 (2015).

[47] P. Y. Wen, A. F. Kockum, H. Ian, J. C. Chen, F. Nori, and I.-C. Hoi, Reflective Amplification without Population Inversion from a Strongly Driven Superconducting Qubit, Phys. Rev. Lett. 120, 063603 (2018).

[48] P. Y. Wen, K.-T. Lin, A. F. Kockum, B. Suri, H. Ian, J. C. Chen, S. Y. Mao, C. C. Chiu, P. Delsing, F. Nori, G.-D. Lin, and I.-C. Hoi, Large Collective Lamb Shift of Two Distant Superconducting Artificial Atoms, Phys. Rev. Lett. 123, 233602 (2019).

[49] Z. H. Peng, S. E. de Graaf, J. S. Tsai, and O. V. Astafiev, Tuneable on-demand single-photon source in the microwave range, Nat. Commun. 7, 12588 (2016).

[50] P. Forn-Díaz, C. W. Warren, C. W. S. Chang, A. M. Vadiraj, and C. M. Wilson, On-demand microwave generator of shaped single photons, Phys. Rev. Appl. 8, 054015 (2017).

[51] Y. Lu, A. Bengtsson, J. J. Burnett, E. Wiegand, B. Suri, P. Krantz, A. F. Roudsari, A. F. Kockum, S. Gasparinetti, G. Johansson, and P. Delsing, Characterizing decoherence rates of a superconducting qubit by direct microwave scattering, npj Quantum Inf. 7, 35 (2021).

[52] U. Dorner and P. Zoller, Laser-driven atoms in half-cavities, Phys. Rev. A 66, 023816 (2002).

[53] L. Guo, A. Grimsmo, A. F. Kockum, M. Pletyukhov, and G. Johansson, Giant acoustic atom: A single quantum system with a deterministic time delay, Phys. Rev. A 95, 053821 (2017).

[54] M. Bradford and J.-T. Shen, Spontaneous emission in cavity QED with a terminated waveguide, Phys. Rev. A 87, 063830 (2013).
[55] A. L. Grimsmo, Time-Delayed Quantum Feedback Control, Phys. Rev. Lett. 115, 060402 (2015).

[56] H. Pichler and P. Zoller, Photonic Circuits with Time Delays and Quantum Feedback, Phys. Rev. Lett. 116, 093601 (2016).

[57] H. Pichler, S. Choi, P. Zoller, and M. D. Lukin, Universal photonic quantum computation via time-delayed feedback, Proc. Natl. Acad. Sci. USA 114, 11362 (2017).

[58] P.-O. Guimond, M. Pletyukhov, H. Pichler, and P. Zoller, Delayed coherent quantum feedback from a scattering theory and a matrix product state perspective, Quantum Sci. Technol. 2, 044012 (2017).

[59] M. P. Schneider, T. Sproll, C. Stawiarski, P. Schmitteckert, and K. Busch, Green's-function formalism for waveguide QED applications, Phys. Rev. A 93, 013828 (2016).

[60] A. González-Tudela and J. I. Cirac, Markovian and nonMarkovian dynamics of quantum emitters coupled to twodimensional structured reservoirs, Phys. Rev. A 96, 043811 (2017).

[61] E. Wiegand, B. Rousseaux, and G. Johansson, Semiclassical analysis of dark-state transient dynamics in waveguide circuit QED, Phys. Rev. A 101, 033801 (2020).

[62] L. Guo, A. F. Kockum, F. Marquardt, and G. Johansson, Oscillating bound states for a giant atom, Phys. Rev. Res. 2, 043014 (2020).

[63] A. Ask, M. Ekström, P. Delsing, and G. Johansson, Cavity-free vacuum-Rabi splitting in circuit quantum acoustodynamics, Phys. Rev. A 99, 013840 (2019).

[64] G. Hétet, L. Slodička, M. Hennrich, and R. Blatt, Single Atom as a Mirror of an Optical Cavity, Phys. Rev. Lett. 107, 133002 (2011).

[65] M. Mirhosseini, E. Kim, X. Zhang, A. Sipahigil, P. B Dieterle, A. J. Keller, A. Asenjo-Garcia, D. E. Chang, and O. Painter, Cavity quantum electrodynamics with atom-like mirrors, Nature (London) 569, 692 (2019).

[66] B. Yurke and J. S. Denker, Quantum network theory, Phys. Rev. A 29, 1419 (1984).

[67] M. H. Devoret, Quantum fluctuations in electrical circuits, in Quantum Fluctuations, Les Houches Session LXIII (Elsevier, Amsterdam, 1995), p. 351.

[68] B. Peropadre, J. Lindkvist, I.-C. Hoi, C. M. Wilson, J. J. GarciaRipoll, P. Delsing, and G. Johansson, Scattering of coherent states on a single artificial atom, New J. Phys. 15, 035009 (2013).

[69] M. Malekakhlagh, A. Petrescu, and H. E. Türeci, CutoffFree Circuit Quantum Electrodynamics, Phys. Rev. Lett. 119, 073601 (2017).

[70] M. F. Gely, A. Parra-Rodriguez, D. Bothner, Y. M. Blanter, S. J. Bosman, E. Solano, and G. A. Steele, Convergence of the multimode quantum Rabi model of circuit quantum electrodynamics, Phys. Rev. B 95, 245115 (2017). 